\title{
An innovative neutron spectrometer for soil moisture measurements
}

 \\ ${ }_{1}^{1}$ Politecnico di Milano- Dipartimento di Energia, Via Labmruschini 4, 20156 Milano, Italy \\ 2 Institut für Kernphysik, J. W. Goethe-Univeristät Frankfurt, Max-von-Laue-Str.1, 60348 Frankfurt am Main, \\ Germany
}

Received: 25 June 2021 / Accepted: 19 September 2021

(C) The Author(s) 2021

\begin{abstract}
Soil moisture is a crucial variable in evaluating soil properties and its interaction with the atmosphere, yet none of the techniques currently employed is fully adequate for evaluating the water content in soil over an area of hectares and depth of tens of centimeters. In recent times, it has been shown how the water content over this volume can be accurately assessed measuring changes in the epithermal flux of cosmic neutrons, which is extremely sensitive to the moderation caused by hydrogen. The instruments employed for neutron flux measurements are usually neutron counters, covered with moderator coatings for enhancing their sensitivity in the epithermal energy range. On the other hand, the worldwide shortage of ${ }^{3} \mathrm{He}$ caused a considerable increase in the costs associated with the manufacturing of proportional counters based on this gas, which were widely employed for their great sensitivity and noise rejection capability. In this work, we developed a ${ }^{3} \mathrm{He}$-free neutron spectrometer for performing these measurements, which detects neutrons in the energy range from 0.01 ev to $1 \mathrm{GeV}$. The reconstruction of the energy spectrum allows a more accurate evaluation of the epithermal neutron flux and provides other information which improves the quality of soil moisture measurements. Irradiations performed with neutron sources of ${ }^{241} \mathrm{Am}$ and AmBe allowed to evaluate the spectrometric capability of the instrument, whereas the measurements of cosmic neutrons were employed to assess its sensitivity to cosmic radiation. The sensitivity of the instrument is slightly less than the one of the neutron counters currently employed, yet the access to the spectrometric information should provide greater accuracy in the epithermal flux measurements.
\end{abstract}

\section{Introduction}

Soil moisture plays a crucial role in controlling the chemical and mechanical properties of a soil, affects the biochemical processes taking place between the land and the atmosphere, moderates the climate of a region, and reflects changes in the water cycle. Nowadays, soil moisture measurements mainly rely on point measurements and satellite remote sensing. The firsts provide accurate estimations of the water content at a single point but are not representative of the surroundings due to the extreme heterogeneity of the water distribution in soil. The latter averages the water content over wide areas (up to tens of thousands of

a e-mail: andrea.cirillo@polimi.it (corresponding author) 




Fig. 1 Cosmic Neutrons Energy Spectrum, from [3]. From the left: the gray region represents thermal neutrons at equilibrium with the environment, the blue region represents the epithermal neutrons, the green region represents the fast neutrons generated by evaporation processes both in the atmosphere and the soil, and the red region represents high energy neutrons generated in the upper atmosphere

square kilometers), suffers a shallow penetration depth $(\mathrm{mm})$, and is sensitive to surface roughness. None of these systems gives a satisfactory measurement on the intermediate scale (i.e., on an area of hectares and a depth of tens of $\mathrm{cm}$ ) [1]. The knowledge of soil moisture in this gap left uncovered by current technology is crucial for applications such as farming and irrigation, as well as to validate models employed in weather, climate, and hydrological forecasts. Nuclear techniques based on cosmic neutron measurements can provide a reliable, relatively inexpensive, and non-invasive way to investigate the water content at this scale [2].

Earth is constantly subjected to a flux of charged particles (mainly protons) coming from our galaxy which interact with the planet atmosphere generating neutrons that are detectable at the ground level. Figure 1 shows the shape of the energy distribution of cosmic neutrons and the regions in which it is conventionally divided. The primary protons interact with oxygen and nitrogen in the atmosphere via intranuclear cascades. As a result, some secondary particles, including high energy neutrons, are generated (red peak in Fig. 1). The resulting nucleus is left in an excited state, from which it can de-excite emitting neutrons in an isotropic process called evaporation. This process can also be triggered in air or soil by a high energy neutron penetrating a nucleus and exciting it to an unstable energy level. In both cases, neutrons with energy of some $\mathrm{MeV}$ are emitted (green peak in Fig. 1). The neutrons produced in these ways are slowed down via elastic collision (blue region in Fig. 1) and eventually reach equilibrium with the surroundings (gray peak in Fig. 1).

In the epithermal and fast region of the energy spectrum $(0.5 \mathrm{eV}-20 \mathrm{MeV})$, neutrons mainly interact via elastic scattering with surrounding nuclei. In this energy range, they become extremely sensitive to hydrogen, which is the most effective neutron scatterer both in terms of interaction probability and energy lost in each collision. Since this element is found in nature as water for the greatest part, the intensity of the epithermal and fast neutron flux is an excellent probe for measuring changes in the level of soil moisture or other water pools (see Fig. 2). Furthermore, since water dominates the physics interactions between neutrons and soil even in concentrations as low as $0.02 \mathrm{~cm}^{3} \mathrm{~cm}^{-3}$, these probes are almost insensitive to any element other than hydrogen.

The soil moisture content can be related to the epithermal neutron flux via the "universal calibration function" [2]: this function features three coefficients which are dictated by nuclear 
Fig. 2 The effect of water content in soil $(\theta)$ on the cosmic neutrons energy spectrum, from [3]. Dry soils show greater fluxes especially in the epithermal $\left(10^{-6}\right.$ to $\left.10^{-1} \mathrm{MeV}\right)$ and fast $\left(10^{-1}\right.$ to $\left.20 \mathrm{Mev}\right)$ energy ranges





Fig. 3 Shape of the universal calibration function. The mathematical expression is given by $\theta\left[\frac{g_{\text {water }}}{g_{\text {soil }}}\right]=$ $\frac{a_{0}}{\phi / \phi_{0}-a_{1}}-a_{2}$, where $\theta$ is the gravimetric soil moisture, $\phi$ is the epithermal neutron flux, $a_{0}, a_{1}, a_{2}$ are three parameters dictated by nuclear physics, and $\phi_{0}$ is the calibration parameter (i.e., the measured flux corresponding to a known value of $\theta$ )

physics and do not depend on the soil chemical composition. The measurement requires a single calibration to assess one calibration parameter and account for stationary water sources in the sensitive area (e.g., water in organic matter, proximal coastlines,...). Figure 3 gives more details about the shape of this function and the above-mentioned parameters.

This method has been successfully employed in USA and Germany, achieving good sensitivity (around $0.01 \mathrm{~cm}^{3} \mathrm{~cm}^{-3}$ ), radial footprints of hundreds of meters, and penetration depths of tens of centimeters (also according to the soil moisture content) [2]. 
Some examples of instruments measuring the neutron flux are ${ }^{3} \mathrm{He}$ proportional counters (widely employed in the USA [2]) and large-scale Boron-lined proportional counters (in Germany [4]). ${ }^{3} \mathrm{He}$ detectors provide a satisfactory sensitivity to neutrons and excellent properties in terms of photon rejection but are generally avoided due to the expense related to ${ }^{3}$ He provisioning. Boron-lined proportional counters are an effective replacement since they can achieve even greater sensitivity to neutrons (at the expense of a less compact design) and a satisfactory noise rejection employing techniques based on the analysis of the shape and amplitude of the recorded pulse. In both cases, these detectors are covered with moderator layers to maximize their sensitivity only to epithermal neutrons, yet they suffer a non-negligible contamination from fast and-more importantly-thermal neutrons. The information coming from neutrons in the non-epithermal region is considered as a contamination for the following reasons:

- The thermal part of the spectrum carries information concerning the soil and vegetation nearby but is highly affected by the environment chemical composition. No "universal calibration function" can correlate this part of the spectrum with the water content alone.

- The fast region carries pretty much the same information as the epithermal one, but with a different calibration function, because fast neutrons are generated both in the atmosphere and in the soil. Some of the neutrons generated in the atmosphere might be detected before having interacted with the soil, which means they are completely insensitive to the water content on the land.

We developed a neutron spectrometer which can be successfully employed for these measurements. The knowledge of the energy spectrum improves the quality of soil moisture measurements for the following reasons:

- The information about high energy neutrons allows real-time, accurate correction for the oscillation of the incoming primary neutron flux.

- The detector can truly isolate the epithermal region of the spectrum, without suffering contamination from thermal and fast neutrons.

- The knowledge of thermal and fast neutron flux can be employed for extracting additional information. Specifically, the thermal region of the spectrum carries information about the changes in the chemical composition of the soil and vegetation, while the fast one is still sensitive to water content in the soil, thus giving information about soil moisture with a shorter radial footprint and penetration depth.

\section{Materials and methods}

\subsection{The detector}

The developed spectrometer is based on the M800 thermal nuclear detector manufactured by Arktis. This detector exploits the neutron reaction of ${ }^{6} \mathrm{Li}$ and the scintillation mechanism of ${ }^{4} \mathrm{He}$. The gas is sealed in a cylinder $\emptyset 14 \times 80 \mathrm{~cm}$, and the lithium is provided in the form of a $\mathrm{LiF}$ thin layer arranged in a way to maximize the surface exposed to the neutron flux. The tube is divided into eight sectors which can be read independently by 24 SiPMs (one triplet per sector). The instrument is commercially available and easy to handle (it does not require high voltage, is power supplied by POE, and IP addressable). A detailed explanation of the detector working principle and the outputs produced can be found in [5].

The detector is divided into four regions (constituted by two adjacent sectors), and each one of them is covered with different coatings to maximize its sensitivity to neutrons in a 


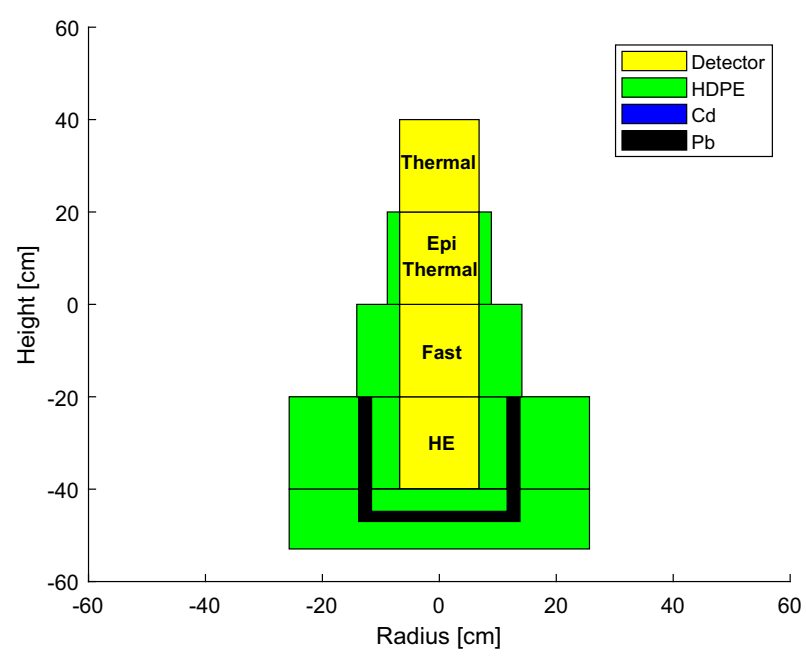

Fig. 4 Coatings for the four regions. The thermal region is bare. The epithermal region is covered with a 2-cm layer of HDPE (high-density polyethylene). The fast region is covered with a 7.2-cm layer of HDPE. The high energy region features the following layers (from the inner to the outer part): (1) $4.8 \mathrm{~cm}$ of HDPE; (2) $0.2 \mathrm{~cm}$ of cadmium; (3) $2 \mathrm{~cm}$ of lead; (4) $5.9 \mathrm{~cm}$ of HDPE. The cadmium layer is too thin to be appreciated in the figure. The inelastic reactions occurring in $\mathrm{Pb}$ allow the detection of neutrons with energy above $20 \mathrm{MeV}$

certain energy range. Having four regions sensitive to different parts of the spectrum allows to perform an unfolding procedure and find the actual neutron spectrum in the point where the detector is placed (whereas the instruments mentioned before only measure the neutron flux in a limited energy range). A representation of the coatings is given in Fig. 4.

The advantages of this solution are several:

1. A single, low-volume, commercially available detector is employed to measure the entire energy spectrum.

2. The solution is cheaper than the ${ }^{3} \mathrm{He}$ detectors and comparable to the boron-lined detector.

3 . The instrument sensitivity and photon rejection capability allow collecting a reliable counting statistic in a few hours.

\subsection{The unfolding algorithm}

The evaluation of the neutron spectrum starting from the counts recorded by the detector requires solving the unfolding problem, which is expressed by Eq. 1.

$$
\mathbf{N}=\mathbf{R} \Phi
$$

$\mathbf{N}$ is the vector containing the counts per second recorded in each region of the detector, $\mathbf{R}$ is the matrix containing the response functions for each region (which were determined via Monte Carlo simulations, see Sect. 2.3), and $\Phi$ is the energy-binned neutron flux. In our case, four regions with different response functions were considered and the energy interval of interest (1E-08 - 1E+03 MeV) was subdivided into 110 bins (10 bins per order of magnitude). It follows that $\mathbf{N}$ is a four-element vector, $\mathbf{R}$ a $110 \times 4$ matrix, and $\Phi$ a 110 - element vector.

The unfolding problem has been tackled by the GRAVEL [6] unfolding algorithm. GRAVEL belongs to the class of nonlinear square gradient methods, and it is employed for its robustness and fast convergence despite being relatively easy to implement [7]. At the 
$K$-th step the algorithm first computes the set of weight $W_{i j}^{(K)}$ using the formula

$$
W_{i j}^{(K)}=\frac{R_{i j} \Phi_{j}}{\sum_{l} R_{i l} \Phi_{l}} .
$$

Then the $\mathrm{K}+1$ tentative spectrum is computed as

$$
\Phi_{j}^{(K+1)}=\Phi_{j}^{(K)} \exp \left[\frac{\sum_{i} \rho_{i} W_{i j}^{(K)} \ln \left(\frac{N_{i}}{\sum_{l} R_{i l} \Phi_{l}}\right)}{\sum_{i} \rho_{i} W_{i j}^{(K)}}\right]
$$

where $\rho_{i}$ is the $i$-th relative variance on the counts $\rho_{i}=N_{i}^{2} / \sigma_{i}^{2}$. Since we assume a Poisson distribution for $N_{i}$, then $\rho_{i}=N_{i}$. The first guess of the spectrum form $\Phi^{(0)}$ must be provided as the initial condition; thus, the convergence speed of the algorithm can be affected by an unhappy choice of the initial guess spectrum. In practice, the guess spectrum must be as close as possible to the expected one and must be chosen exploiting the a priori information about the physic of process of neutron generation. In addition, being the correction at each step multiplicative, a null guess in an energy bin cannot be modified by the GRAVEL algorithm.

Being a least-square like method, GRAVEL iterates until $\chi^{2}=\sum_{i} \sigma_{i}^{-2}\left(\sum_{j} R_{i j} \Phi_{j}-N_{i}\right)^{2}$ is reduced below a user-defined threshold. This is of particular interest in practical applications since the user can tune the $\chi^{2}$ parameter to have a fast simulation for a gross estimate of the spectrum or a slower but much more precise estimation. The algorithm has been tested on tabulated data and implemented in Python3.

\subsection{Monte Carlo simulations}

Monte Carlo simulations were mainly performed for assessing the response to neutrons of the regions in which the detector is divided, as well as to optimize the moderators' thicknesses for easing the unfolding procedure as much as possible.

The assessment of the detector response relies on simulations performed via the MCNPX ver 5.1 code. MCNP is a general-purpose Monte Carlo N-Particle code that can be used for neutron, photon, electron, or coupled neutron/photon/electron transport [8].

A complete modeling of the detector is hard to achieve since the manufacturer keeps confidentiality about the exact content of ${ }^{6} \mathrm{Li}$. On the other hand, the detector data sheet states that $1.05 \mathrm{cps}$ (counts per second) are recorded when the instrument is placed in a highdensity polyethylene (HPDE) box of known dimensions and irradiated with $1 \mathrm{ng}$ of ${ }^{252} \mathrm{Cf}$ at a $2 \mathrm{~m}$ distance. The first simulations represented the detector in the geometry indicated by the data sheet, and the ${ }^{6} \mathrm{Li}$ content was tuned according to the nominal sensitivity. A precise measurement of the instrument sensitivity in reference neutron fields will be the object of future work.

Once the detector was reasonably modeled, the full geometry sketched in Fig. 4 was included in the simulation to assess the instrument response function in the neutron energy range $0.01 \mathrm{eV}-1 \mathrm{GeV}$.

The source was represented by an expanded aligned field, and the response function was given in terms of cps per unit neutron flux. The coatings' thicknesses were optimized to maximize the sensitivity of each region to neutrons in a limited energy range. Figure 5 shows the response function for each instrument region. It is worth noting that the absolute content of ${ }^{6} \mathrm{Li}$ does not affect the shape of the response functions, as long as it is homogeneously distributed over the regions. This last condition is experimentally verified because the bare detector (i.e., without any coating) shows statistically compatible count for each sector. Even 


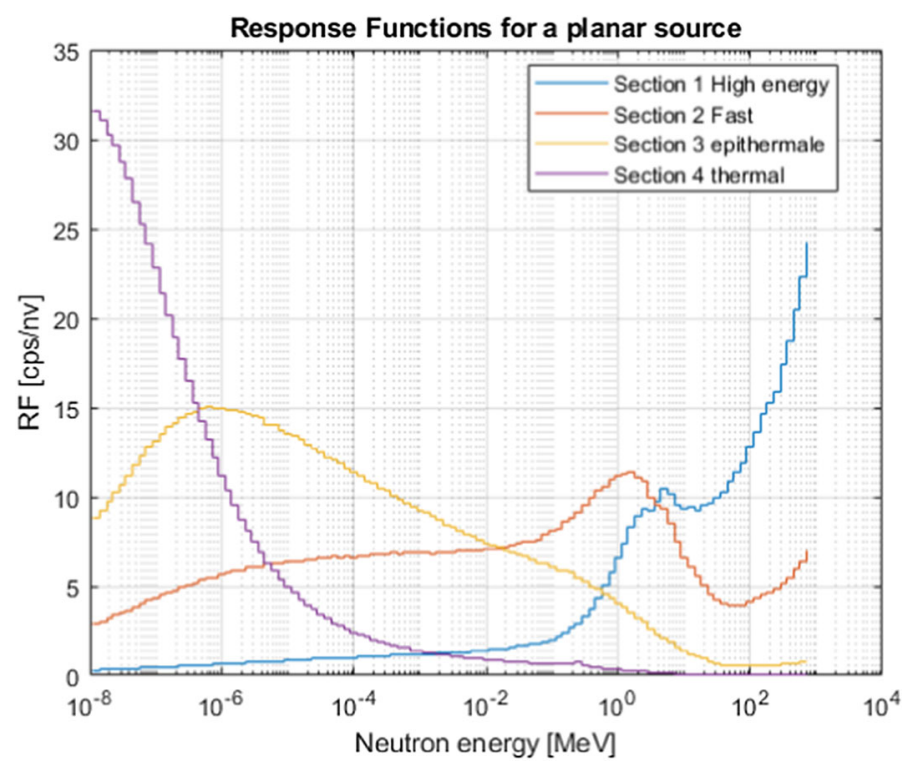

Fig. 5 Response functions for the four regions. The ordinate axis shows the cps per unit flux $\left(\frac{c p s}{n v}\right)$ as a function of the neutron energy. The responses are found in case of an expanded aligned neutron field

if the ${ }^{6} \mathrm{Li}$ content estimated by the previous simulations was not precise, the four response functions would vary by the same factor. In that case, the unfolding procedure represents correctly the energy distribution, despite scaling by a constant factor the value of the neutron flux. This aspect does not penalize soil moisture measurements since the integrals of the thermal, epithermal, and fast neutron regions are normalized to the integral of the high energy neutron region, which is representative of the source term (i.e., the total cosmic neutron flux).

\section{Results}

Three different measurements were taken to assess the spectrometer capability to reconstruct the neutron energy distribution:

1. The detector was irradiated with an AmBe source;

2. The detector was irradiated with an Am source;

3. The detector was placed on the forecourt and the roof of the B 18 building of the Department of Energy of Politecnico di Milano, to assess its response to cosmic neutrons.

The results are given hereafter. As the instrument has been conceived as an extended range spectrometer to measure cosmic neutrons, the moderators have been designed with this goal in mind. As a consequence, the response matrix is not optimized for the emission spectrum from Am and AmBe.

\subsection{AmBe irradiation}

For the first irradiation session, the detector was placed at a $2 \mathrm{~m}$ distance from an AmBe source. The irradiation was performed in the Secondary Standard Calibration Laboratory (LAT No. 104) of the Department of Energy of Politecnico di Milano. The Calibration Laboratory has 


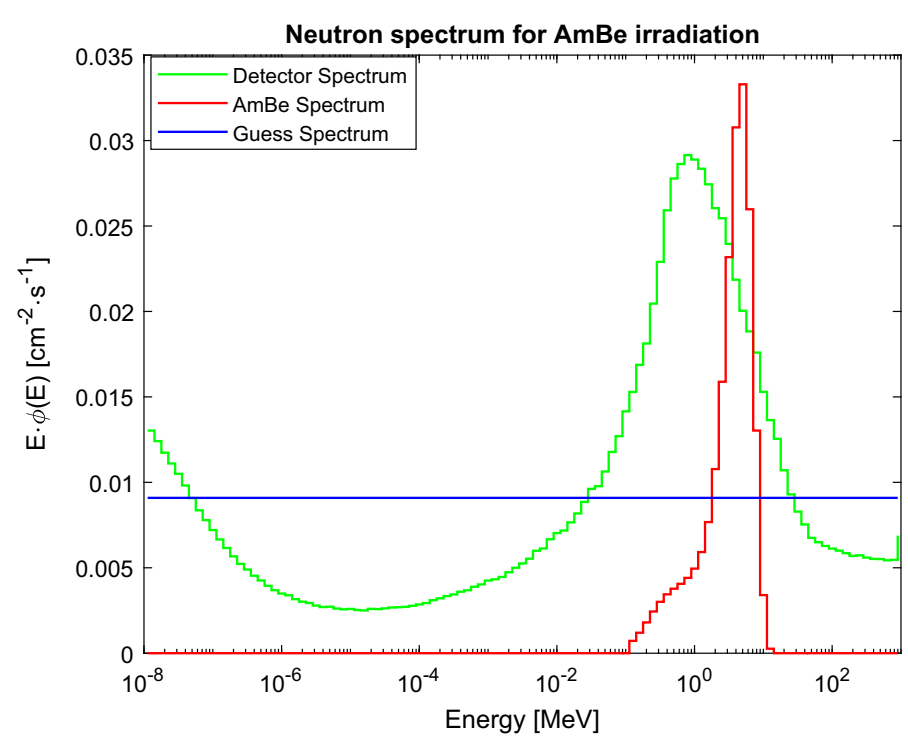

Fig. 6 Reconstructed spectrum (green) and guess spectrum (red) for AmBe irradiation. The reconstructed and guess spectra are normalized for having unitary integral. The AmBe emission is shown in red expressed in arbitrary units for better showing the energy range in which the source emission falls

two irradiation facilities employed for generating photon fields. The dimension of the room in which the test was performed is not optimized for neutron irradiation, and several neutrons undergo scattering inside the room before being detected. As a consequence, the energy spectrum at the point where the detector is placed does not coincide with the one emitted by the source.

Figures 6 and 7 show the spectra calculated by the unfolding algorithm with two different guess spectra (a flat guess in Fig. 6 and a spectrum in which the AmBe emission is visible in Fig. 7).

A second measurement was taken keeping the source in the same position and placing in front of it a water slab phantom [9]. The slab phantom is a water-filled $30 \mathrm{~cm} \times 30 \mathrm{~cm} \times 15 \mathrm{~cm}$ slab, employed as a representation of the human torso for photon dosimeters calibration. Its water content makes it an excellent moderator for fast neutrons.

In Figs. 8 and 9 the spectra obtained for these irradiations were compared with the one calculated for the non-moderated source.

\subsection{Am irradiation}

The detector was irradiated placing at $2 \mathrm{~m}$ distance a $1 \mathrm{Ci}$ Am source, whose $\gamma$ emission was shielded by a 5-cm-thick Pb shutter. The irradiation was performed in the LAT No. 104 (see Sect. 3.1). The Am source is typically used for $\gamma$ irradiations, but due to its chemical form as an oxide, the nuclear reaction ${ }^{18} \mathrm{O}(\alpha, \mathrm{n}){ }^{21} \mathrm{Ne}$ produces a weak neutron emission of about $10^{4} \frac{n}{s}$ over $4 \pi\left({ }^{18} \mathrm{O}\right.$ is an oxygen isotope with a natural abundance of $\left.0.2 \%\right)$. The Americium source neutron emission has already been measured in a previous campaign [5]. Also in this case, the scattering occurring in the room is expected to alter the neutron energy distribution in the point where the detector is placed, which will therefore be different from 


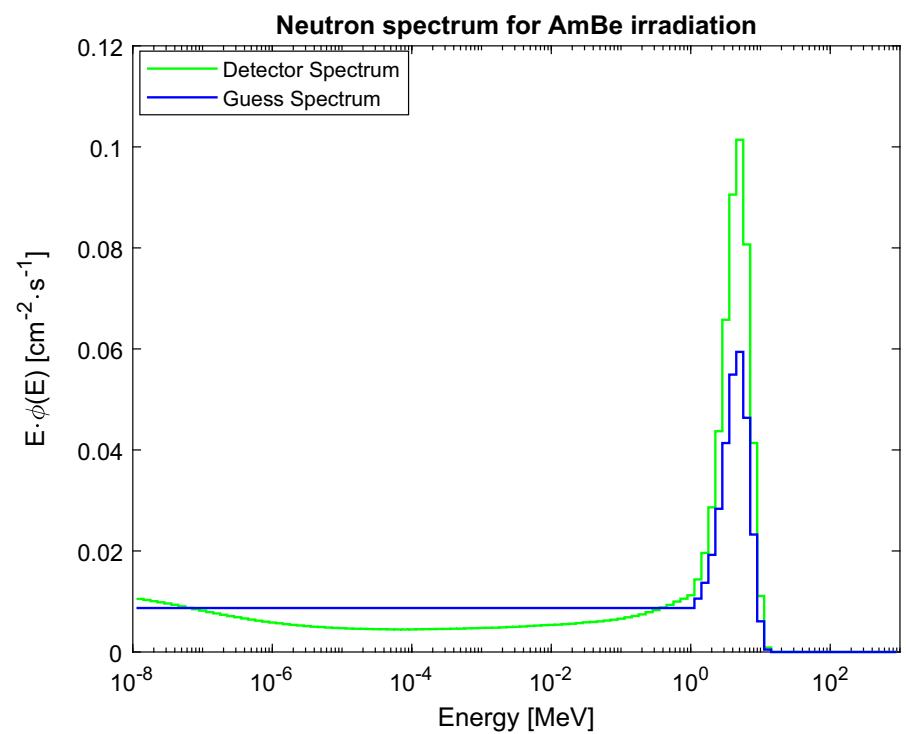

Fig. 7 Reconstructed spectrum (green) and guess spectrum (blue) for AmBe irradiation. The spectra are normalized for having unitary integral



Fig. 8 Comparison between the calculated spectrum for irradiation with a moderated (green, solid line) and non-moderated (green, dashed line) AmBe source, starting from a flat guess spectrum (blue line). The spectra are normalized for having unitary integral. The AmBe emission is shown in red expressed in arbitrary units for better showing the energy range in which the source emission falls 


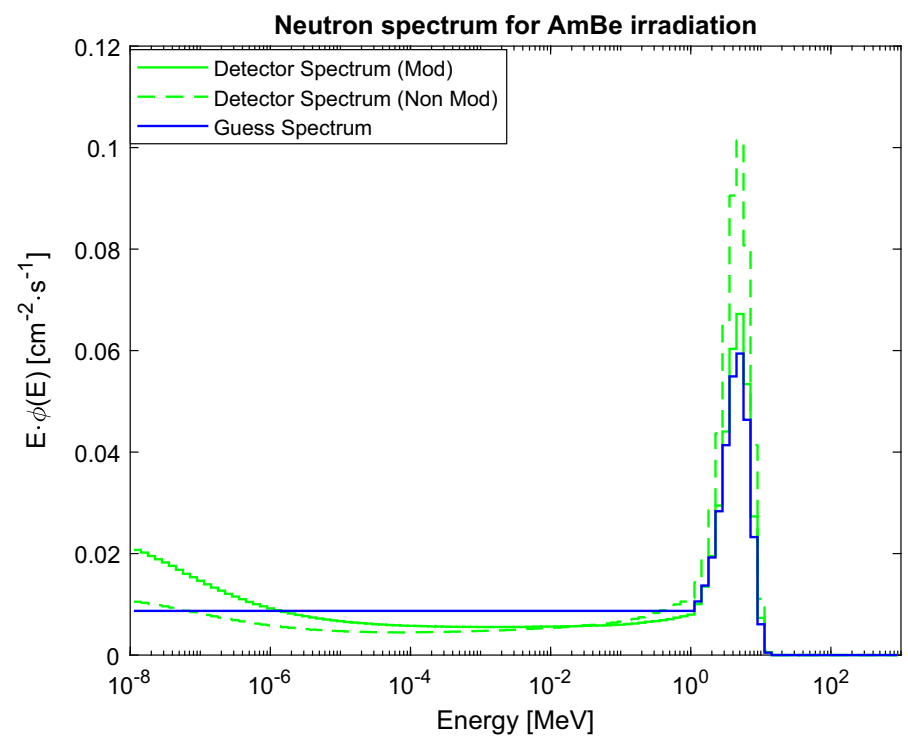

Fig. 9 Calculated spectrum for irradiation with a moderated (green, solid line) and non-moderated (green, dashed line) AmBe source. The guess spectrum is shown by the blue line. Spectra are normalized for having unitary integral

the one emitted by the source. Figure 10 shows the calculated energy spectrum when a flat guess was given to the unfolding code.

\subsection{Cosmic Neutron irradiation}

Two measurements were taken to assess the instrument capability of detecting cosmic neutrons. First, the detector was placed on the forecourt a few meters far from the B18 building of the Department of Energy of Politecnico di Milano (15 $\mathrm{m}$ tall); then, the measurement was repeated on the roof of the same building (around $12 \mathrm{~m}$ above ground). Figure 11 shows the spectra obtained in these conditions. The counts per second (cps) recorded by each region of the instrument are reported in Table 1.

\section{Discussion}

The measurements taken with neutron sources proved the capability of the system of fairly recognizing their energy distribution. In Figs. 6 and 10 the fast component of the spectrum is identified, even though the guess spectra give no indication about the energy range in which the source emission falls. In addition, it is worth noting that in soil moisture measurements the detector only needs to classify the neutron energy in four regions: thermal, epithermal, fast, and above $20 \mathrm{MeV}$. As long as the neutron count is placed in the right region, the exact value of the energy peak has little influence on the measurement accuracy.

As expected, a realistic guess spectrum results in a fairly accurate output spectrum. In Fig. 7 the detector shows the peak associated with the AmBe emission, as well as the thermalized component of the spectrum, which was not introduced in the starting guess spectrum. 


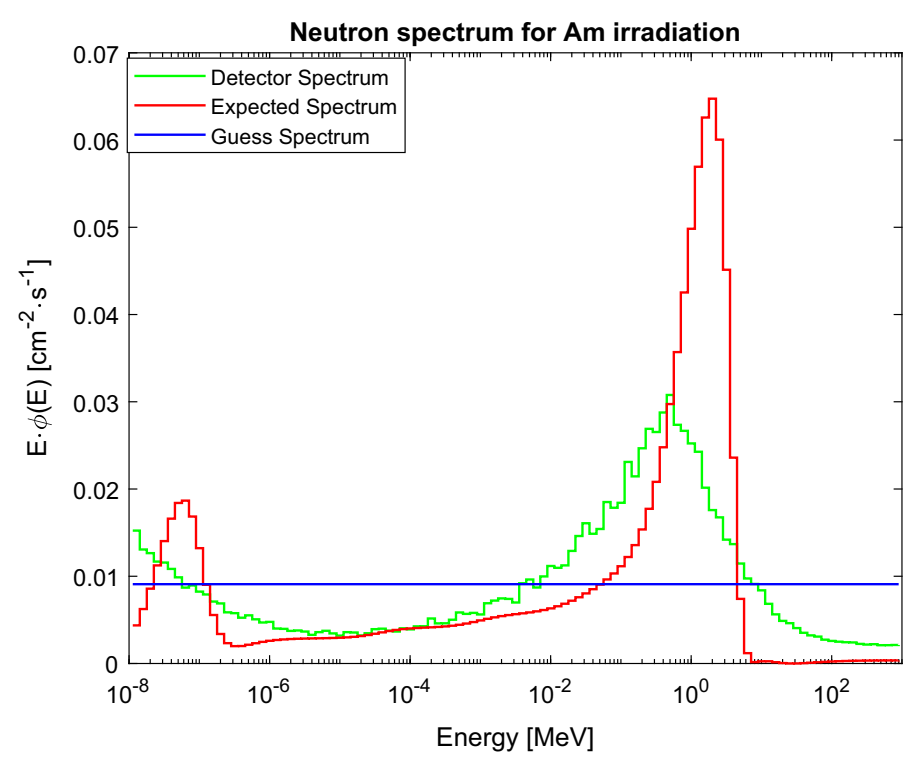

Fig. 10 Calculated spectrum for the Am source (green line), starting from a flat guess spectra (blue line). The spectra are normalized for having unitary integral. The expected Americium spectrum (red line) shows the peak associated with the energy of neutrons emitted after the ${ }^{18} \mathrm{O}(\alpha, \mathrm{n}){ }^{21} \mathrm{Ne}$ reaction and the one associated with the thermalized component

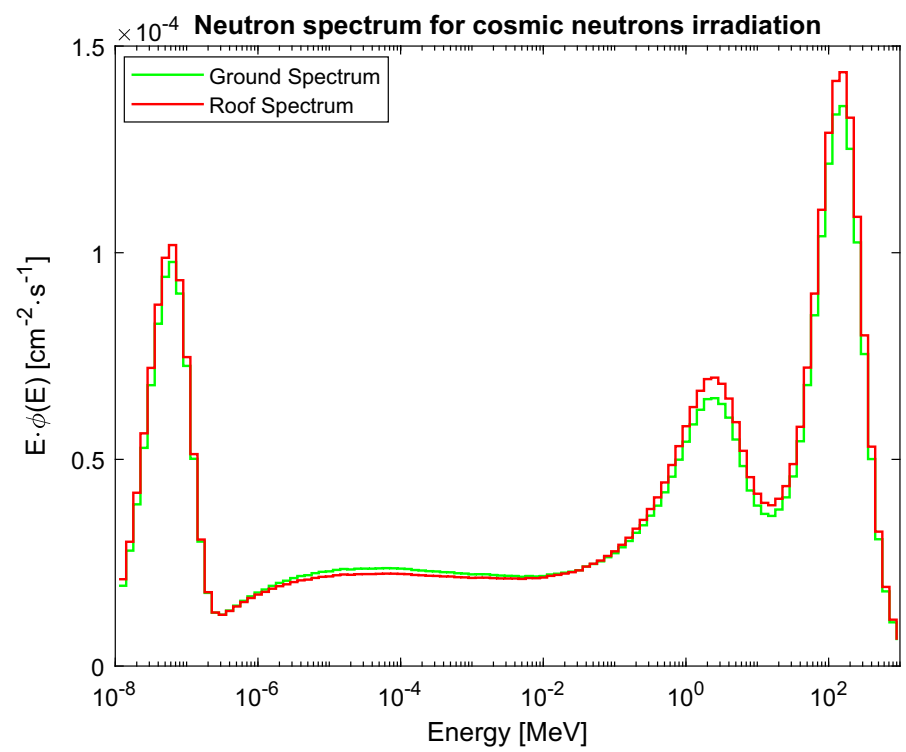

Fig. 11 Reconstructed neutron spectra for the ground (green line) and roof (red line) level 


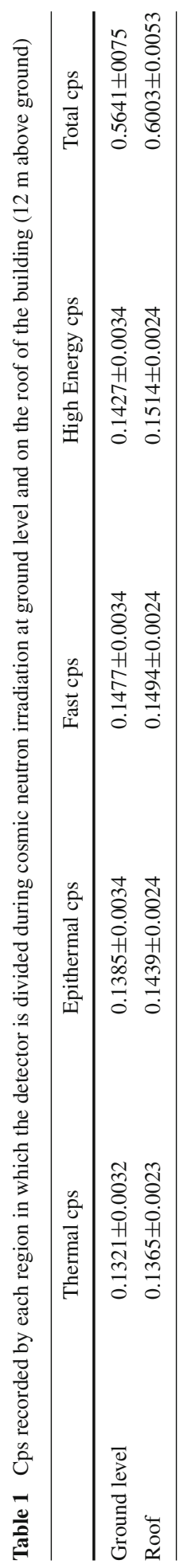


In addition, the tests performed with the moderated AmBe source (Figs. 8 and 9) showed the detector capability of identifying the changes in the neutron spectrum when the neutron emission is moderated, without changing the guess spectrum. This aspect is crucial since soil moisture measurements rely on the evolution of the epithermal (and possibly fast) component of the spectrum due to changes in the moderating power of the soil.

The two measurements of cosmic neutrons were aimed at assessing the instrument capability of acquiring satisfactory statistics in reasonable time and fairly reproducing the neutron energy distribution on the surface. This distribution is indeed complex (it is hard to believe that the instrument could reproduce its main components starting from a flat guess spectrum), but it is relatively well known and reproduced even via mathematical models. Because of this, the unfolding procedure started from a reasonable neutron spectrum calculated by the algorithm PARMA[10], introducing the latitude, longitude, and altitude of Milan as calculation parameters.

Figure 11 shows the spectra measured at the roof and ground level. The differences in the region for $\mathrm{E} \leq 20 \mathrm{MeV}$ are at least partially ascribable to the differences in the chemistry of the region surrounding the detector. On the other hand, the decrease in the high energy peak (i.e., for $E \geq 20 \mathrm{MeV}$ ) entirely depends on the attenuation of the primary neutron beam, which is caused by the air column above the detector and, more importantly, by the buildings close to the measurement point. In both experiments, the detector was not placed in a truly open field, and the buildings in the surroundings unavoidably suppressed the incoming neutron flux. This effect is definitely more severe at ground level than in an elevated position.

The last aspect that the cosmic neutron measurements were aimed to assess was the counting statistic. Soil moisture measurements clearly benefit from high count rates, which minimize the Poisson uncertainty associated with the events recorded. Moderated ${ }^{3} \mathrm{He}$ detectors employed for performing these measurements are mainly sensitive to the epithermal region of the neutron spectrum and collect roughly 1000 counts per hour (cph), which leads to an uncertainty of some $\%$ in water concentration. Our instrument recorded $500 \mathrm{cph}$ in the epithermal region. On the other hand, some aspects must be considered when evaluating the system response:

- This count rate was measured in an urban environment in which the incoming cosmic flux is suppressed and is expected to be an underestimation of the value which would be recorded in an open field.

- These counts are not directly related to the epithermal neutron flux, which is estimated after performing the unfolding algorithm.

Concerning the last aspect, the unfolding algorithm is based on an iterative procedure which complicates a mathematical evaluation of the uncertainty of the neutron flux. The uncertainty of the epithermal component of the spectrum was estimated with the following procedure:

1. The cps in Table 1 ("Roof" row) were considered as the real interaction rate for each region of the detector

2. A counting time was chosen

3. The expected counts in each region were calculated as Counts $=$ cps $\times$ counting time, and each of them was considered Poisson distributed

4. Four new values of counts (one for each region) were sampled from as many Poisson distributions

5. The unfolding was run and the epithermal neutron flux was calculated

6. The previous steps were repeated until 10000 values of epithermal neutron flux were obtained 
Fig. 12 Distribution of the values of epithermal neutron flux calculated by the unfolding algorithm varying the counts recorded in each region. The uncertainty of the flux value scales down approximately with the square root of the counting time



(a) One hour

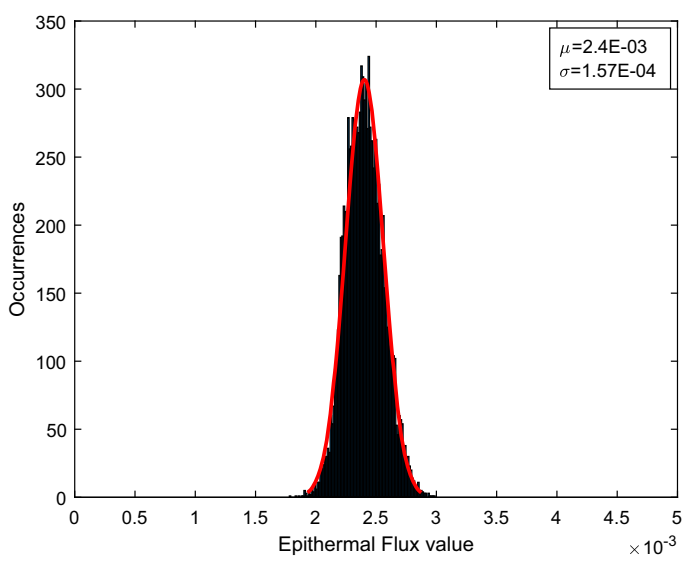

(b) Two hours

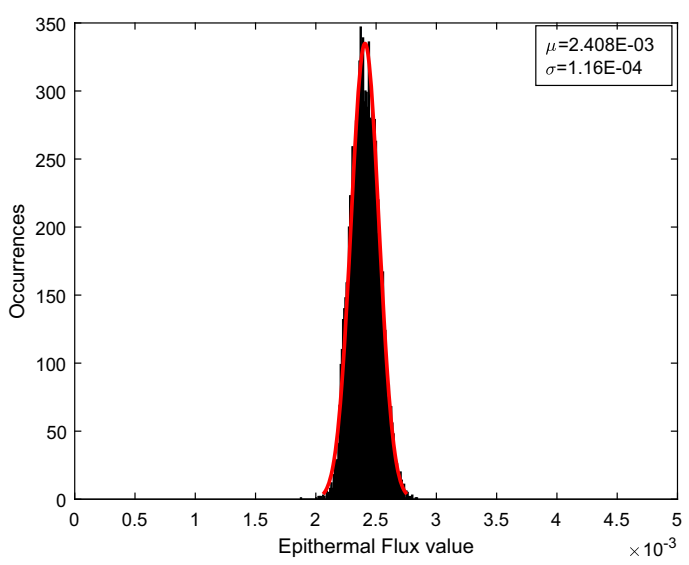

(c) Four hours 
Table 2 Relative uncertainty associated with the epithermal neutron flux for different counting times

\begin{tabular}{ll}
\hline Counting time $(\mathrm{h})$ & $\sigma(\%)$ \\
\hline 1 & 8.9 \\
2 & 6.5 \\
4 & 4.8 \\
\hline
\end{tabular}

7. The set of epithermal neutron fluxes was fitted by a Gaussian function, and the mean and variance were estimated.

Figure 12 shows the obtained results for the epithermal neutron flux measured at the roof level, considering counting times of 1,2, and $4 \mathrm{~h}$. Table 2 shows how the relative uncertainty is affected by the counting time. As expected, the uncertainty scales down approximately with the square root of the counting time. For the count rates measured on the roof of the building, a counting time of four hours must be chosen if the uncertainty on the epithermal neutron flux has to be kept below $5 \%$.

Considering the one-hour measurement, 500 counts are expected to be recorded in the epithermal region of the detector. The Poisson uncertainty associated with them is $4.5 \%$, about one-half of the uncertainty reported in Table 2. On this basis, it might appear that the unfolding procedure worsens the precision of soil moisture measurements. Nevertheless, it must be considered that the epithermal region of the detector shows a non-null sensitivity to thermal and fast neutrons (see Fig. 5). Such neutrons' contribution increases the counts recorded by the detector and decreases the Poisson uncertainty, yet it is not related to the water content in the soil via the universal calibration function. This means that the thermal and fast neutrons measured "contaminate" the signal, making the soil moisture assessment less accurate. The spectrometer, on the other hand, discards this contamination employing the unfolding algorithm and isolates the epithermal region of the spectrum. It can be imagined as if the instrument could selectively detect epithermal neutrons. The counts associated with this component of the spectrum are less than the ones effectively recorded (which depend also on the thermal and fast contamination) and are therefore associated with a higher uncertainty.

\section{Conclusions}

In this paper, we tested an innovative neutron spectrometer, specifically aimed to measure the cosmic neutron energy distribution and relate the epithermal neutron flux to the water content in soil. The M800 detector manufactured by Arktis was divided into four regions covered with different moderator layers, the response function of each region was assessed via Monte Carlo simulations, and an unfolding algorithm implemented in Python3 allowed the real-time reconstruction of the neutron spectrum.

To begin, the detector was tested employing neutron sources (one AmBe and one Am). In both cases, the instrument successfully recognizes the fast emission of the source, even when the starting guess spectrum was perfectly flat. In addition, it is sensitive to the moderation of the source emission. On the other hand, when the unfolding algorithm is provided with a more realistic distribution, a finer reconstruction of the spectrum is obtained.

The detector also measured the natural radiation both at ground level and in an elevated position. These measurements were mainly aimed to test its sensitivity to cosmic neutrons: in this configuration, the detector counted roughly $0.6 \mathrm{cps}$ (summing all regions). This value 
is the starting point for assessing the uncertainty of the neutron flux in the energy region of interest.

It was estimated that, after performing the unfolding algorithm, an uncertainty of $9 \%$ is associated with the epithermal flux for measurements lasting one hour, which decreases to $5 \%$ if the counting time is raised to four hours. This value might appear poor when compared to the relative uncertainties of the count rates for several neutron counters employed, yet it must be noticed that these counters are sensitive, to some extent, to thermal and fast neutrons, which contaminate the soil moisture assessment since they are not related to the water content by the same calibration function. A neutron spectrometer, on the other hand, can truly isolate the epithermal component of the spectrum, giving a more accurate estimation of the water concentration in soil.

This work proved the instrument capability of performing reliable neutron spectrometry. More research will investigate its performance in assessing the water content in soil and the benefits of employing a complete spectrometer instead of a neutron counter sensitive to the epithermal energy range.

Acknowledgements The authors gratefully acknowledge ARKTIS Radiation Detectors Company for providing the M800 employed for developing the instrument. We would also like to show our gratitude to ELSE NUCLEAR S.r.l. and Officina Meccanica Finotti Sergio for their contribution in the manufacture of the coatings employed.

Funding Open access funding provided by Politecnico di Milano within the CRUI-CARE Agreement.

\section{Declarations}

Funding Politecnico di Milano provided Open Access funding within the CRUI-CARE Agreement.

Conflicts of interests The authors have no conflicts of interest to declare that are relevant to the content of this article.

Availability of data and material The datasets generated during and/or analyzed during the current study are available from the corresponding author on reasonable request.

Open Access This article is licensed under a Creative Commons Attribution 4.0 International License, which permits use, sharing, adaptation, distribution and reproduction in any medium or format, as long as you give appropriate credit to the original author(s) and the source, provide a link to the Creative Commons licence, and indicate if changes were made. The images or other third party material in this article are included in the article's Creative Commons licence, unless indicated otherwise in a credit line to the material. If material is not included in the article's Creative Commons licence and your intended use is not permitted by statutory regulation or exceeds the permitted use, you will need to obtain permission directly from the copyright holder. To view a copy of this licence, visit http://creativecommons.org/licenses/by/4.0/.

\section{References}

1. D.A. Robinson, C.S. Campbell, J.W. Hopmans, B.K. Hornbuckle, S.B. Jones, R. Knight, F. Ogden, J. Selker, O. Wendroth, Soil moisture measurement for ecological and hydrological watershed-scale observatories: A review. Vadose Zone J. 7(1), 358-389 (2008)

2. M. Zreda, W.J. Shuttleworth, X. Zeng, C. Zweck, D. Desilets, T. Franz, R. Rosolem, Cosmos: the cosmicray soil moisture observing system. Hydrol. Earth Syst. Sci. 16(11), 4079-4099 (2012)

3. M. Köhli, M. Schrön, M. Zreda, U. Schmidt, P. Dietrich, S. Zacharias, Footprint characteristics revised for field-scale soil moisture monitoring with cosmic-ray neutrons. Water Resour. Res. 51(7), 5772-5790 (2015)

4. J. Weimar, M. Köhli, C. Budach, U. Schmidt, Large-scale boron-lined neutron detection systems as a $3 \mathrm{He}$ alternative for cosmic ray neutron sensing. Front. Water 2, 16 (2020) 
5. G. Zorloni, P. Tancioni, M. Caresana, Feasibility study of a shielding-independent radiation portal monitor system for revealing 241-am orphan sources in radiometric surveillance of scrap metal. Eur. Phys. J. Plus 135, 703 (2020)

6. M. Matzke, Unfolding procedures. Radiat. Prot. Dosim. 107(1-3), 149-168 (2003)

7. Y.H. Chen, X.M. Chen, J.R. Lei, L. An, X.D. Zhang, J.X. Shao, P. Zheng, X.H. Wang, Unfolding the fast neutron spectra of a bc501a liquid scintillation detector using gravel method. Sci. China Phys. Mech. Astron. 57(10), 1885-1890 (2014)

8. G. Mckinney. Mcnpx user's manual, version 2.6.0, 04 (2008)

9. Report 39. J. Int. Comm. Radiat. Units Meas. os 20(2):NP-NP (2016)

10. T. Sato, H. Yasuda, K. Niita, A. Endo, L. Sihver, Development of parma: Phits-based analytical radiation model in the atmosphere. Radiat. Res. 170, 244-259 (2008) 